\title{
Uso de acondicionamento ambiental para matrizes suínas em gestação
}

Patrícia de SOUSA ${ }^{1}$; Irenilza de Alencar NÄ̈̈S

Correspondência para: IRENILZA DE ALENCAR NÄÄS

Faculdade de Engenharia Agrícola Unicamp

Caixa Postal 6011

13083-970 - Campinas - SP

irenilza@agr.unicamp.br

Recebido para publicação: 23/09/2003 Aprovado para publicação: 15/03/2004

\author{
1 - Faculdade de Engenharia Agrícola da UNICAMP, Campinas - SP
}

\author{
Palavras-chave: \\ Ambiência. \\ Gestação. \\ Resfriamento. \\ Adiabático. \\ Suínos.
} reprodutivo. O objetivo deste trabalho foi avaliar o efeito do resfriamento adiabático associado à ventilação controlada (SRAVC), utilizado no alojamento de matrizes suínas gestantes, no seu desempenho reprodutivo. Foram comparados dois tipos de sistemas SRVC e ventilação natural (Natural). Um total de 144 matrizes Dalland ${ }^{\circledR}$ foram alojadas em duas diferentes estações, verão e inverno, resultando em 4 tratamentos (33 matrizes cada): sistema controlado com ventilação forçada e nebulização durante o verão (SRAVC/Verão); sistema de ventilação natural durante o verão (Natural/Verão); sistema controlado com ventilação forçada e nebulização durante o inverno (SRAVC/Inverno) e sistema de ventilação natural durante o inverno (Natural/Inverno). O sistema era regulado a partir de um termostato onde os ventiladores eram acionados a uma temperatura ambiente de $25^{\circ} \mathrm{C}$ e a nebulização a $27^{\circ} \mathrm{C}$, permanecendo ligada por cinco minutos e desligada durante 20 minutos. Foram avaliados os seguintes parâmetros produtivos: número de leitões nascidos vivos $(\mathrm{NV})$, número de mumificados $(\mathrm{MF})$, número de natimortos $(\mathrm{NM})$, peso médio ao nascer (PN), número de leitões desmamados (NLD), peso médio ao desmame (PD) e ganho de peso dos leitões (GP). O sistema SRAVC proporcionou a redução do número de leitões mumificados no plantel, enquanto a estação do ano contribuiu para melhorar no peso ao desmame dos leitões.

\section{Introdução}

O suíno é um exemplo de animal cujo conforto vem sendo alterado pela intensificação da produção, caracterizada pela restrição do espaço, movimentação e interação social ${ }^{1,2}$ o que traz como conseqüência secundária o detrimento de seu conforto térmico, assim como da sua produtividade. A determinação das exigências de bem-estar animal em relação à saúde e à economicidade da produção, constitui um grande desafio para a simplificação do manejo, redução de custos e aumento da produtividade ${ }^{3}$.

As respostas de animais domésticos ao micro-ambiente a que estão expostos têm sido estudadas intensivamente, no sentido de se entender o funcionamento dos mecanismos homeostáticos ${ }^{4,5,6,7}$. Este conhecimento tem servido de base para as decisões modernas de controles ambientais em sistemas intensivos de produção animal, pelo entendimento das respostas fisiológicas destes. Entretanto, ainda são pouco conhecidas respostas que permitam decisões em tempo real, uma vez que esta base de dados refere-se principalmente a animais dos anos 80 , que diferem geneticamente dos utilizados em produções comerciais hoje.

O desempenho produtivo e reprodutivo dos suínos depende do sistema de manejo, que envolve o sistema de criação, da nutrição, da sanidade e das instalações. Estas instalações, maior volume de investimento inicial fixo, são construídas em função dos custos e 
facilidades para o tratador, ficando geralmente negligenciado o conforto do animal. Alguns autores ${ }^{8,9,10,11,12}$ estudaram as relações térmicas dos materiais de construção com a ventilação das edificações para abrigo de animais, bem como o efeito da radiação solar nos cálculos das cargas térmicas e sugerem que, ao controlar-se os efeitos da radiação solar direta e indireta, elimina-se grande parte dos problemas de excessiva carga térmica na instalação.

$\mathrm{Nas}$ regiões tropicais, as perdas registradas na suinocultura, nas várias fases de produção, onde a maioria das instalações é inadequada às condições climáticas, ocorrem devido ao desconhecimento dos princípios de ambiência pelos técnicos do setor. A produção máxima é limitada pelas altas temperaturas associadas aos altos índices de umidade relativa. A instalação zootécnica deve visar o controle de elementos climáticos, como a temperatura, umidade relativa, ventilação, insolação, além de higiene, alimentação e bem-estar, que possibilitam o conforto térmico, pois, segundo a categoria animal, a produção será favorecida numa determinada condição do ambiente ${ }^{13}$. As variações ambientais são controladas com diferentes materiais de construção, dimensionamento da baia, densidade e sistema de ventilação ${ }^{14}$.

Altas temperaturas podem afetar todos os estágios, desde o desenvolvimento da puberdade até a reprodução e nascimento dos leitões 9 . Há publicado hoje, um vasto conhecimento acumulado sobre a influência da incidência de altas temperaturas no desempenho de suínos, $4,7,8,15,16,17$, entretanto, as alternativas propostas advêm, em sua grande maioria, de soluções apropriadas para climas temperados. Alguns dos efeitos negativos do clima em matrizes gestantes são: atraso no desenvolvimento normal dos níveis de hormônios em marrãs, redução na taxa de concepção, aumento na mortalidade ao nascimento, aumento na incidência de aborto e mortalidade embrionária ${ }^{18}$.

Pesquisas recentes ${ }^{19,20}$ encontraram eficiência das técnicas de resfriamento em suínos em vários estágios de produção, quando submetidos ao estresse calórico. $\mathrm{O}$ resfriamento adiabático resultante da existência de área úmida ao redor dos animais alojados, aumenta a sensação de conforto térmico, assim como reduz a temperatura do local em cerca de $3^{\circ} \mathrm{C}$. Quando essas técnicas de resfriamento estão associadas a um correto sistema de ventilação, os resultados podem ser potencializados ${ }^{21}$. O efeito de ambiente climático adequado ao animal, talvez não se reflita em melhora significativa na produção, pois há fatores como a genética, a nutrição e a sanidade do rebanho a serem considerados. A sinergia desses fatores permite a continuidade de estudos nesta área, dada a dificuldade de se isolar os fatores que atuam nesse sistema dinâmico ${ }^{22}$.

Tendo em vista a necessidade de se conhecer soluções adequadas às condições tropicais de alojamento de matrizes gestantes, foi objetivo deste trabalho avaliar o efeito do resfriamento adiabático com utilização de ventilação associada a nebulização controlada (SRAVC), sobre o desempenho reprodutivo de matrizes gestantes.

\section{Materiais e Métodos}

O experimento foi realizado em uma granja comercial localizada no município de Campinas. A classificação climática do local segundo Koppen, é clima subtropical, seco no inverno com temperatura média anual de $24,5^{\circ} \mathrm{C}$ e precipitação média anual de $1360 \mathrm{~mm}$. A latitude local é $22^{\circ} 54^{\prime} \mathrm{S}$, a longitude $47^{\circ} 05 \mathrm{~N}$ e a altitude 674 metros. O galpão de gestação onde foram colhidos os dados possuía as seguintes características construtivas: orientação Leste - Oeste; dimensões de $50 \mathrm{~m}$ de comprimento por $10 \mathrm{~m}$ de largura; parede aberta nas laterais com ventilação natural; pé-direito de $2,5 \mathrm{~m}$; beiral de $0,8 \mathrm{~cm}$; cobertura com telhas de fibrocimento pintadas de branco na parte interna e externa (cal na parte interna e cal + fixador na parte externa); piso e parede de 
alvenaria.

O período de colheita de dados foi de dezembro de 1998 a dezembro de 2001. Foram avaliados dois tipos de sistemas: ventilação natural e ventilação controlada associada a nebulização de alta pressão temporizada. Foram utilizadas 36 matrizes (raça Dalland ${ }^{\circledR}$ e multíparas, entre o terceiro e o oitavo parto) por tratamento (Natural e $S R A V C$ ) em cada estação (verão e inverno), totalizando 144 fêmeas no experimento, com quatro tratamentos: 1 - Sistema controlado com ventilação forçada e nebulização com alta pressão e temporização durante o verão (SRAVC/verão); 2 - Sistema de ventilação natural durante o verão (Natural/verão); 3 - Sistema controlado com ventilação forçada e nebulização com alta pressão e temporização durante o inverno (SRAVC/inverno); 4 - Sistema de ventilação natural durante o inverno (Natural/inverno).

O sistema SRAVC caracterizou-se pela utilização de dois ventiladores em direções opostas e uma linha de nebulização de alta pressão, com 10 bicos de pulverização. O sistema foi ajustado a partir de um termostato onde os ventiladores eram acionados aos $25^{\circ} \mathrm{C}$ e a nebulização aos $27^{\circ} \mathrm{C}$, sendo que este, permanecia ligado por cinco minutos e, posteriormente, desligado durante 20 minutos. Os dados ambientais foram registrados dentro da instalação: temperatura de bulbo seco (Tbs), temperatura de bulbo úmido (Tbu) e temperatura de globo negro (Tgn). Para o registro das medidas de Tbs, Tbu e $\operatorname{Tgn}\left({ }^{\circ} \mathrm{C}\right)$ foram utilizados Dataloggers individuais da marca DIDAI ${ }^{\circledR}$, que registraram dados a cada 30 minutos, durante todo o período experimental.

Foram avaliados os efeitos dos tratamentos durante a fase de gestação de matrizes suínas com relação aos seguintes parâmetros produtivos: número de leitões nascidos vivos (NV); número de mumificados (MF); número de natimortos (NM); peso médio da leitegada ao nascer (PN), número de leitões desmamados (NLD), peso médio dos leitões ao desmame
(PD) e ganho de peso dos leitões (GP). Os dados de produção foram analisados após o desmame dos animais.

Inicialmente foi averiguada a diferença estatística entre os dados de clima dos anos de experimento, não sendo detectada diferença pelo teste Tukey $(\alpha=$ $0,05)$. As análises estatísticas dos dados foram realizadas pelo programa computacional Minitab $^{\circledR}$, em fatorial $2 \times 2$, sendo dois fatores (sistema e estação) e dois níveis cada (natural e SRAVC; verão e inverno).

O modelo estatístico utilizado na análise dos dados foi o seguinte:

Yijk $=+\alpha i($ sistema $)+\beta j($ estação $)+(\alpha \times \beta) i j+\xi_{i j k}$

Eq. (1)

$(i=0-1 ; j=0-1)$.

Onde;

Yijk - variável resposta (NV, MF, NM, PN, NLD, PD, GP);

$\mu$ - média geral de Yijk;

$\alpha i$ - efeito do nível i do fator "sistema" sobre a média geral do modelo (sendo: $\mathrm{i}=0$ natural, ou $\mathrm{i}=1$ SRAVC);

$\beta j$ - efeito do nível $j$ do fator "estação" sobre a média geral do modelo (sendo: $\mathrm{i}=0$ natural, ou $\mathrm{i}=1 \mathrm{SRAVC}$ );

$(\alpha x \beta)$ ij - Interação do nível i do fator "sistema" e o nível j do fator "estação" do modelo; $\xi_{\text {ij }}$ - Erro independente das variáveis aleatórias com distribuição normal $(0, \sigma 2)$.

\section{Resultados}

As médias de temperatura de bulbo seco, bulbo úmido e globo negro, assim com as amplitudes térmicas calculadas, durante o período de verão, estão sumarizadas na tabela 1.

Os valores absolutos das amplitudes térmicas durante o verão e inverno foram de $19,1^{\circ} \mathrm{C}$ e $14,3^{\circ} \mathrm{C}$, respectivamente. Dos parâmetros de desempenho medidos e avaliados, apenas dois foram significativos em nível de $\alpha=0,01$, o número de leitões mumificados (MF) e o peso ao desmame 
Tabela 1-Valores de Tbs, Tgn e Tbu do ambiente interno durante o verão e o inverno

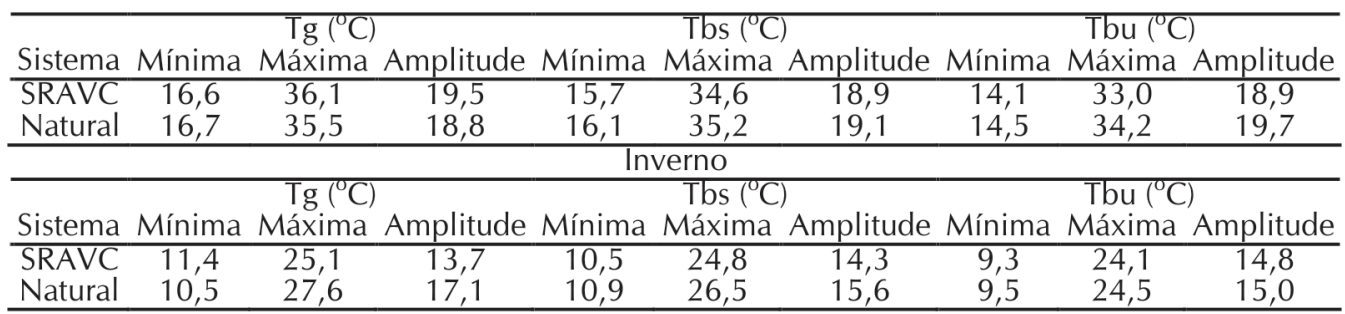

Tabela 2 - Médias de leitões mumificados (MF) e peso ao desmame dos leitões (PMD)

\begin{tabular}{ccc}
\hline Tratamento \Parâmetro & MF & PMD (kg) \\
\hline SRAVC \Verão & $1,47 b$ & $5,86 b$ \\
Natural \Verão & $1,70 \mathrm{a}$ & $5,90 \mathrm{~b}$ \\
SRAVC $\backslash$ Inverno & $1,30 \mathrm{c}$ & $5,93 \mathrm{a}$ \\
Natural \Inverno & $1,48 \mathrm{~b}$ & $6,03 \mathrm{a}$ \\
\hline
\end{tabular}

\section{Discussão e Conclusões}

O comportamento do clima local foi constante, não apresentando anormalidades que comprometessem os resultados do experimento. Não houve diferença $(\alpha=0,01)$ nos resultados de temperatura nem entre as amplitudes térmicas dentro da instalação, considerados os sistemas estudados. Diferentes destes resultados, Turner et al. ${ }^{19}$ obtiveram resultados que mostraram a redução significativa de Tbs e da amplitude térmica, com a adição de ventilação associada a nebulização, em instalações abertas para suínos. Não foi também identificada correlação entre as características físicas do ambiente construído e os sistemas testados, de maneira que os resultados dos dados de ambiente foram resultantes do uso dos sistemas utilizados, diferente do que preconizam Clark ${ }^{9}$ e Henken et al. ${ }^{11}$. Isto ocorreu principalmente pela presença das aberturas laterais que permitiram fluxo de ar externo constante e, com isso, o sistema influenciou o ambiente interno, quando justamente havia calor excessivo associado à falta de ventilação externa, coincidindo com o encontrado por Curtis ${ }^{4}$ e Geers et al. ${ }^{17}$.
O sistema de resfriamento adiabático com ventilação controlada proporcionou a redução do número de leitões mumificados no plantel, tanto no verão como no inverno (Tabela 2). Pedersen ${ }^{8}$ encontrou aumento de produtividade em leitões terminados submetidos a ventilação forçada, enquanto Turner et al. ${ }^{19}$ encontraram que o desempenho de suínos aumentou significantemente com a adição de ventilação associada a nebulização, em instalações abertas. Comparando ventilação natural com o túnel de vento (pressão negativa) em galpões de terminação, Lally e Edwards ${ }^{20}$ determinaram que os animais alojados em edificação com túnel de vento tiveram maiores taxas de conversão alimentar do que aqueles alojados com ventilação natural, demonstrando o benefício do conforto térmico no desempenho produtivo. $\mathrm{O}$ melhor resultado de MF no tratamento SRAVC, pode estar relacionado com o fornecimento de boas condições de conforto térmico às matrizes alojadas nas baias individuais, durante as primeiras semanas de gestação, como sugere Clark?.

Piva ${ }^{23}$ também analisou o estresse de calor em matrizes em gestação e concluiu que, em desconforto térmico, as fêmeas produzem alto nível de absorção e de número de leitões mumificados. Enquanto Banhazi et al. ${ }^{24}$ encontraram que, matrizes gestantes suínas submetidas a condições ótimas de conforto convertem mais precisamente o alimento em produção de leite, desmamando leitegadas mais pesadas. No presente trabalho, a diferença encontrada foi referente à estação (inverno e verão), independente do sistema adotado.

O sistema de resfriamento adiabático 
com ventilação controlada influenciou positivamente na redução do número de leitões mumificados, supostamente explicado pelo conforto térmico fornecido às matrizes na fase de gestação, especificamente na fase antes do parto, não modificando o desenvolvimento dos leitões. Concluiu-se que o uso do resfriamento adiabático com ventilação controlada contribuiu somente para melhorar o número de leitões mumificados, tendo a estação do ano influenciado no peso ao desmame dos leitões.

\section{Agradecimentos}

Os autores agradecem à FAPESP, pelo apoio financeiro ao trabalho e à Granja Marilena, por ceder as instalações e animais utilizados no experimento.

\section{Environmental modification for gestating sows}

\section{Abstract}

The effect of high environmental temperature on animals has been extensively studied in order to search the ideal housing and better performance both productive and reproductive. The objective of this research was to evaluate the effect of the evaporative cooling system associated to controlled ventilation (SRAVC) used in gestating sow's housing on their reproductive parameters response. Two systems were evaluated: SRAVC and natural ventilation (Natural). A total of 144 Dalland ${ }^{\circledR}$ sows were used during two seasons, summer and winter, resulting in 4 treatments (33 sows each) such as: the use of evaporative cooling associated to controlled ventilation during summer (SRAVC/ summer), natural ventilation during summer (Natural/summer), evaporative cooling associated to controlled ventilation during winter (SRAVCwinter) and natural ventilation during winter (Natural/ winter). The SRAVC system control was adjusted by thermostat, and the ventilation started when the ambient temperature reached $25^{\circ} \mathrm{C}$, and fogging at $27^{\circ} \mathrm{C}$, keeping it on for five minutes and then turning it off for the next twenty minutes. The following reproductive parameters were evaluated: number of piglets born alive (NV); mortality of embryo (MF), mortality at birth (NM), weight at birth $(\mathrm{PN})$, number weaned piglet (NLD), weaned piglet's weight (PD), and piglet's weight gain (GP). The forced ventilation system decreased the embryo mortality, but the only the season influenced the final weight of weaned piglets.

\section{Referências}

1 VAN PUTEN, G. The pig: Model for discussing animal behavior and welfare. Applied Animal Behavior Science, Amsterdan, v. 22, p. 115-128, 1989.

2 HIGGS, G.; SENIOR, M.; WILLIAMS H. Spatial and temporal variation of mortality and deprivation. Widening health inequalities. Environment and Planning, v. 30, p. 1661-1682, 1998.

3 KOLACZ, R.; DOBRZANSKI, Z. Thermoregulatory reponse and activity of AspAt, A1AT and creatine kinase inpregnant sows during overheating stress. Medycyna
Key-words:

Environment.

Gestating.

Evaporative Cooling.

Sows.
- Weterynaryjna, v. 43, n. 12, p. 745-748, 1987.

4 CURTIS, S. E. Environmental management in animal agriculture. lowa: lowa State University Press, 1983. $563 \mathrm{p}$.

5 VERSTEGEN, M. W. A. et al. Influence of some environmental, animal and feeding factors on energy metabolism in growing pigs. In: VERSTEGEN, M. W. A.; HENKEN, A. M.; NIJHOFF, M.; DORDRECHT, N. L. (Ed.). Energy metabolism of farm animals. 1987. p. 70-86.

6 ALBRIGHT, L. D. Environmental control for animals 
and plants. St. Joseph, MI: ASAE, 1990. 213 p.

7 WATHES, C. M. Environmental control in pig housing. In: INTERNATIONAL PIG VETERINARY SOCIETY CONGRESS, Birmingham, UK. 15., 1998, Proceedings... Nottinghan: Nottinghan University Press, 1998. v. 1, p. 257-265.

8 PEDERSEN, S. Optimal temperature and air velocity in houses for fattening pigs. In: AUMAITRE, A.; LE DIVIDICH, J.; TEXIER, P. (Ed.). Housing and climatic environmental for pig. INRA: Paris, France, 1980. $p$ $37-49$

9 CLARK, J. A. Enviromental aspects of housing for animal production. London: Butterworths, 1981. 234 p.

10 GHELFI FILHO, H.; SILVA, I. J. O.; MOURA, D. J.; Consigliero, F. R. Índice de conforto térmico e CTR para diferentes materiais de cobertura em três estações do ano. In: CONGRESSO BRASILEIRO DE ENGENHARIA AGRÍCOLA, 1991, Londrina-PR. Anais... SBEA: Londrina-PR, 1992. p 94-110.

11 HENKEN A. M. et al. Circadian rhythm in heat production of limit-fed growing pigs of several breeds kept at and below thermal neutrality. Journal of Animal Science, v. 71, p. 434-1440, 1993.

12 HARDOIM, P. C.; LOPES, S. P. Análise comparativa de cinco tipos de materiais de cobertura em condições de temperatura máxima em Lavras. In: CONGRESSO BRASILEIRO DE ENGENHARIA AGRÍCOLA, 1993, Ilhéus, Anais... SBEA: Ilhéus-BA. 1993. p 107-117.

13 PERDOMO, C. C.; NICOLAIEWSKY, S. Comportamento ambiental de diferentes modelos de edificações para suínos, durante a estação quente. Comunicado técnico Embrapa, 1986. p. 1-2.

$14 N \mathrm{~A} A ̈ \mathrm{~S}$, I. A. Princípios do conforto térmico na produção animal. São Paulo: Icone, 1989. 183 p.

15 SAINSBURY, D. W. B. Climatic Environment and pig performance, In: COLE, D. J. A. (Ed). Pig production. London: Butterworths. 1972. p. 91-105.

16 MOUNT, L. E. The assessment of thermal environment in relation to pig production. Livestock Production Science, v. 2, p. 381-392, 1975.

17 GEERS, R. et al. Environmental temperature control by the pigs comfort behavior through image analysis. Transactions of ASAE, v. 34, p. 2583-2586. 1991

18 ASHRAE. Handbook of fundamentals. Atlanta: American Society of Heating and refrigerating and Airconditioning Engineers, 1985. 656 p.
19 TURNER, L. W. et al. Fan, sprinkler, and sprinkler plus fan systems for cooling growing-finishing swine. ASAE: St. Joseph, MI, 1997. ASAE Paper \#974076.

20 LALLY, J. J.; EDWARDS, W. M. Performance differences in swine finishing facilities with natural and tunnel ventilation. Applied Engineering in Agriculture, v. 17 , n. 4 , p. 521-526. 2001.

21 CIGR. Climatization of animal housing. 2 nd. Report of Working Group. Comisssion Internacionale du Génie Rural, 1989. 128 p.

22 MAGHIRANG, R. G.; JEREZ S. B.; PREDICALA B. Z. Relative ventilation effectiveness in a mechanically ventilated airspace under isothermal conditions. Transactions of the ASAE, v. 44, n. 3, p. 691-696, 2001.

23 PIVA, J. H. Aspectos não nutricionais que afetam o desempenho de suínos. Boletim Informativo Agroceres PIC, Patos de Minas, MG, 1993. 109 p.

24 BANHAZI, T. et al. The effects of adverse environmental and drinking water temperatures on pig production. Australia, 2000. 123 p. Final report to PRDC Canberra. 